\title{
Response to: "Critical Analysis of a Hypothesis of the Planetary Tidal Influence on Solar Activity" by S. Poluianov and I. Usoskin
}

\author{
J.A. Abreu • C. Albert • J. Beer • A. Ferriz-Mas • \\ K.G. McCracken · F. Steinhilber
}

Received: 11 November 2013 / Accepted: 2 January 2014 / Published online: 16 January 2014

(C) Springer Science+Business Media Dordrecht 2014

In their analysis of our hypothesis of a planetary influence on solar activity Poluianov and Usoskin $(\mathrm{P} \& \mathrm{U})$ claim that the 'peaks in long-period range of the torque spectrum are artefacts caused by the calculation algorithm' and that 'the statistical significance of the wavelet coherence is overestimated'.

We will show here that the periodicities between 60 and 600 years which we found in the torque exerted by the planets on the tachocline are not artefacts. However, averaging the daily torque data has an effect on the corresponding spectrum. In our paper A12 (Abreu et al., 2012) we did not use wavelet coherence to estimate the statistical significance of the discussed periodicities. Nevertheless, we will address the question of the significance of the periodicities as well.

All existing records of solar activity based on cosmogenic radionuclides have a temporal resolution of one year at best. We therefore decided to average the daily $x, y$, and $z$ components of the torque over one year. This procedure is different from the classical aliasing problem, which occurs by resampling the data in discrete steps of one year, but, as we realized later, it has an effect on the resulting spectrum.

A detailed spectral analysis reveals the following.

J.A. Abreu ( $\varangle) \cdot$ C. Albert · J. Beer · F. Steinhilber

Eawag, Swiss Federal Institute of Aquatic Science and Technology, Postfach 611, 8600 Duebendorf, Switzerland

e-mail: jose.abreu@eawag.ch

A. Ferriz-Mas

Departemento the Física Aplicada, Universidade de Vigo, Vigo, Spain

A. Ferriz-Mas

Instituto de Astrofísica de Andalucía (IAA/CSIC), Granada, Spain

K.G. McCracken

University of Maryland, College Park, USA 
i) All the five periodicities we discuss in A12 are present in the daily torque data. However, their amplitudes are relatively small. In the ranking of the strongest periodicities they show up on positions between 15 and 30 .

ii) As a result of the averaging over one year the five periodicities move up to positions between 1 and 10 .

iii) Averaging over two or more years decreases the amplitudes again.

iv) The reduction of Equations (1) - (3) to (5) - (7) is incorrect and leads to a time series which is different from ours. Setting all the geometrical factors to 1 is equivalent to assuming a spherical tachocline in which case the planetary torque vanishes.

v) We do not know whether the shift of the 506-year peak to 430 years in Figure 3 (top panel), which P\&U consider as 'a minor difference', is the result of this. In any case it conflicts with their Figures 4 and 5 where it appears correctly at about 506 years.

vi) We would like to conclude our comments about artefacts by mentioning that planetary torque requires an amplification mechanism in the Sun in order to modulate the long-term heliomagnetic activity. Whatever this physical mechanism will be, we cannot exclude that it may act as a filter.

In the second part of their paper $P \& U$ criticize our significance analysis. We stress that in our paper we used wavelet techniques only in the context of phase analysis and not for estimating the significance. Figure 6 in $P \& U$ shows the wavelet coherence for two periodicities we do not find in our data. However, the crucial question to be answered is: what is the probability that all five spectral lines are simultaneously present by chance in a time series of the same length as our records of solar activity? Monte Carlo techniques show unambiguously that this probability is lower than $10^{-4}$.

\section{Reference}

Abreu, J.A., Beer, J., Ferriz-Mas, A., McCracken, K.G., Steinhilber, F.: 2012, Is there a planetary influence on solar activity? Astron. Astrophys. 548, A88. 